\title{
Is Astronomical Research Appropriate for Developing Countries?
}

\author{
Michael. S. Snowden \\ P.O. Box 44, MacPherson Rd, Singapore 913402. e-mail: \\ mss@sri.lanka.net
}

\begin{abstract}
An unproductive 45-cm astronomical telescope, given by JICA (Japan) to Sri Lanka, raises general questions as to the reasons for unproductive pure science in developing countries. Before installation, site, maintenance, and scientific objectives were discussed. The facility was launched with a conference organised by the UN Office for Outer Space Affairs. Unfortunately, no research or significant education has resulted after four years. The annual operating cost is U.S. $\$ 5000$ per year, including salary for a trainee, maintenance, and a modest promotional programme. Comparison with a similar installation in Auckland suggests lack of funding or technical competence do not explain the failure in Sri Lanka. The facility in New Zealand, on the roof of Auckland University's Physics Department, has a slightly smaller budget but has led to modest but useful research and teaching. Lack of financial backing and expertise are often blamed for weak science in developing countries, but examination shows most of these countries have adequately skilled people, and plenty of resources for religion and military. General lack of motivation for science appears to be the principal reason. This lack of interest and highly inefficient bureaucracies are common to scientifically unproductive countries. They mostly lack the cultural and philosphical base of the European Renaissance that motivate the pursuit of modern science, an activity that violates human preferences. There are excellent facilities (ESO, SAAO, Cerro Tololo, and GONG) in some of these same countries, when administered from the West.
\end{abstract}

\section{Some Personal Experiences in Sri Lanka}

In 1993 October, I received a phone call from my long-time friend, Sir Arthur Clarke. He asked me if I would attend a Sri Lanka government meeting as his representative, because he himself was unable to attend. The meeting was chaired by K. Austin Perera, the Secretary of the Ministry of Industries, Science and Technology, and was concerned with locating an observing site for a modern $45-\mathrm{cm}$ reflecting telescope and with setting up a related administration. The telescope was being donated by JICA, a government department in Japan that administers aid to Third-World countries. As I am an observational astronomer 
with some experience in the early development of observatories, and as a quasiresident in Sri Lanka for several years, he felt that I might help the committee. I happily agreed and requested that Mr. Simon Tulloch, a visiting astronomer from England, also attend.

The committee was composed of a panel of technical experts in meteorology, computer science, and engineering who were particularly familiar with the practical realities associated with government bureaucracies in Sri Lanka. It was only a matter of minutes before Mr. Tulloch and I saw the same conflicts arising in this meeting that Americans and Europeans had debated and resolved 30 years earlier about locating observatories (Irwin, 1952, 1966, and 1966). The issues at the meeting were mostly ones dealing with convenience of the location versus an effective environment for a telescope, but also ones dealing with administering a modern observatory with a zero-rupee budget.

Somewhat nonplussed, Mr. Tulloch and I heard a quotation by a notable Japanese astronomer, "With modern techniques and detectors you can compensate for a bad location", and the committee added a similar one of its own, "security at a remote location in Sri Lanka would be impossible." In that meeting only Mr. Tulloch and I were motivated to look for a location with clear sky conditions, while the others wanted it located "downtown" for convenience and security as a sort of end in itself. Considering the serious mood, I refrained from quoting for the committee Sir Arthur's celebrated quip about observatory sites, "Big telescopes should be built at bad sites to compensate for the poor conditions." It might have been taken seriously.

On the question of administering the facility, the committee was unanimously skeptical. Not a single rupee had been budgeted for installation or operation, and any institution accepting the facility could be inviting a white elephant.

Mr. Tulloch and I saw two pressing concerns. No one actually knew the sky conditions for astronomical purposes in Sri Lanka, and without some sort of immediate and basic site survey there was a certain disaster in the making. As there were no more than a few old typed comments on musty old paper by Dr. Tom Gehrels in the 1950s about potential sites and some brief suggestions by a local meteorologist, we quickly volunteered for a basic site survey. In retrospect, I know today that our collecting the facts was the last thing that that committee wanted.

For two weeks in 1993 October, Mr. Tulloch and I visited the central highlands of Sri Lanka from Anuradhapura to Samanalawewa. Upon conclusion, we submitted a formal report to the committee, and after my departure, Mr. Tulloch continued the survey alone. He identified several possible and practical sites for situating a small astronomical observatory (Tulloch, 1996). In his recommendations, he noted the particularly useless location being considered on top of the Arthur Clarke Institute for Modern Technologies (ACCIMT) in Moratuwa, where he was employed.

Fortuitously, I encountered a particularly ironic moment during the survey. One evening at Kandy, a substantial Sri Lankan cultural centre, I enjoyed a chance encounter with a prominent senior civil engineer, Dr. Ray Wijewardena, who was actively involved with a government water-management project and who took interest in our site-surveying activities. Upon hearing the full story, 


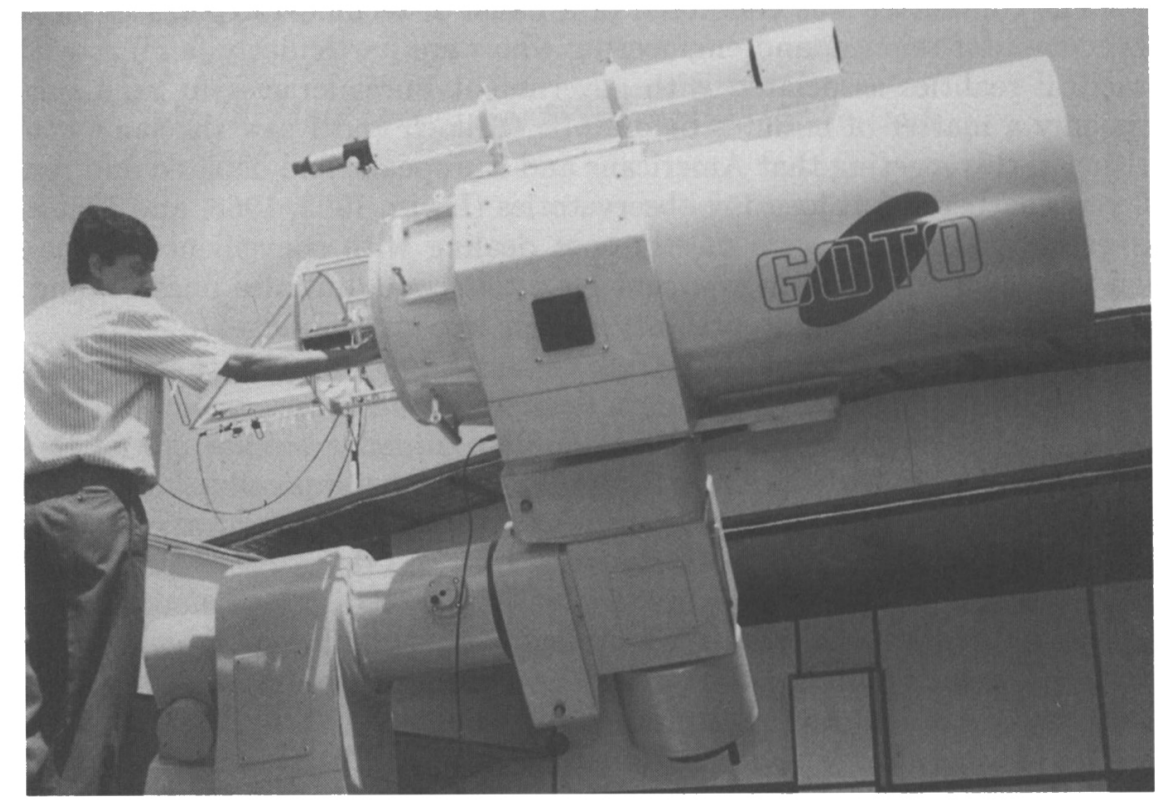

Figure 1. The 45-cm GOTO telescope at the Arthur Clarke Institute (courtesy ACCIMT).

Dr. Wijewardena asserted that an astronomical research observatory was "the last thing this country needs", and he went on to assure me that he would personally see to it that the project would be stopped. He did not. While not quite the response I appreciated at the time, I later came to appreciate his considerable wisdom, which was based on far more Sri Lankan experience than I had had.

All of our suggestions and reports were ignored, and early in 1996 the GOTO Japanese 45-cm telescope was installed, during the UN/ESA "Workshop on Basic Space Science: From Small Telescopes to Space Missions", at the Arthur Clarke Institute, where it is currently located as a white elephant among bright urban lights and in the remains of a tropical coastal rain forest (Figure 1).

The theme of the conference was concerned with the application of small telescopes to current research problems, and experts gave interesting papers in fields germane to this focus. Informally during the meeting, the chairman, Prof. Hans J. Haubold, urged individuals to follow the conference with on-going support for the astronomy programme in Sri Lanka.

The initial cost of the facility was primarily in the staggering $\$ 500,000$ cost of the GOTO telescope plus a rolling roof atop the Arthur Clarke Institute for Modern Technologies. Today, it is maintained there at a cost to the Sri Lankan 
government of $\$ 5000$ per year, which is used for the maintenance of the telescope and building, the salary of one trainee, and a promotional programme.

Although students at the University of Moratuwa have told me that they have not benefitted in any way by the presence of the telescope, and although there is still no instrumentation for the telescope, the facility has enjoyed some significant success in impressing schoolchildren. Japan continues to help the facility occasionally, but as a research tool, the telescope is only draining a painfully poor country of limited resources, just as Dr. Wijewardena predicted. So, there sits a high-quality and unusable $45-\mathrm{cm}$ telescope, which to date has not produced a single datum of meaningful astronomical data, and no research student has benefited by its being there.

\section{A Comparison with the University of Auckland}

In a different corner of the former British Empire, there is a country which has followed twentieth-century development with a different philosophical base from that of Sri Lanka. In New Zealand there is a similar project in progress, and it is one for which a comparative analysis is revealing. At the Physics Department at the University of Auckland in New Zealand, there is a modest astronomical pursuit on the roof top of a building similar to the Arthur Clarke Institute (Figure 2). There, a 35-cm telescope is currently in use every clear night at a dreadful observatory location, which is surrounded by a large city and extensive lighting, but that is the limit of the similarities.

The telescope is used to train about 150 first-year university laboratory students and about 30 stage-three students. The 400 hours/year of observing at Auckland over three years of activity has resulted in data collected for five to ten cataclysmic binaries and about three publications. Standard deviations for the differential time-series CCD photometry is typically $5-9 \mathrm{mmag}$ at $\mathrm{V}=13.0$.

In short, a shoestring budget in New Zealand has produced a modest but real scientific activity which is producing educational and research benefits commensurate with their investment. The success in New Zealand can be attributed to some clear skies compared to almost none at the Sri Lanka site and to a programme in Auckland that includes a good understanding and precise calibrations of their instruments. Most of all, it lies on a foundation which is a long British tradition of science and education. In Sri Lanka an extremely expensive donation and investment has produced almost nothing. With such a striking comparison we are compelled to ask the question in the heading of the next section.

\section{What went Wrong in Sri Lanka?}

Dig deep, and ye shall find! For any institution in a First-World country that is interested in helping an observatory in the Third World, this is an important question, but I have been repeatedly impressed that the answers I have heard are superficial and have missed the point. Whatever the correct answer it does indeed lie deep.

In attempting to install modern pure science into Third-World countries, we are behaving to a considerable extent like Christian missionaries, for better or worse. Should we be doing missionary work? On the other hand, what happens 


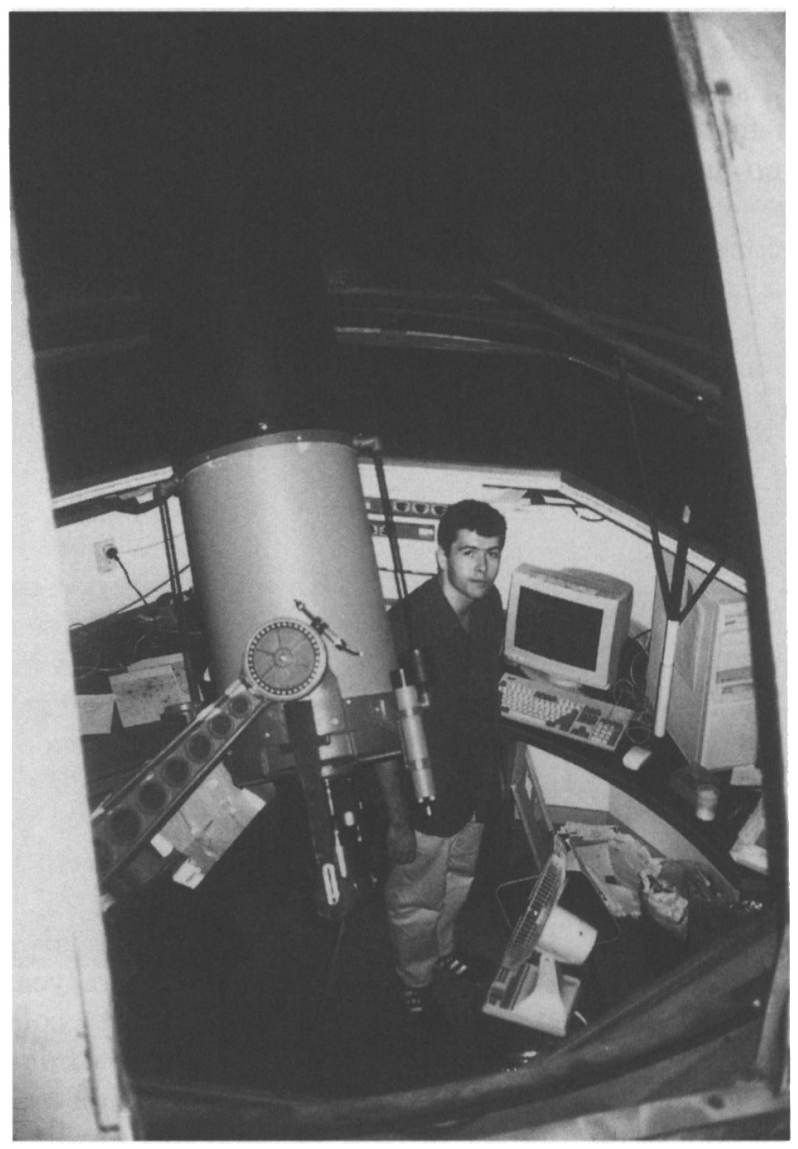

Figure 2. The 35-cm telescope at the Physics Department of Auckland University.

when those in developing countries are left to their own devices in pursuing modern science?

The philosophical base for modern science, i.e., the paradigm currently in practice with the combination of empirical and deductive elements, is inexorably linked to the Renaissance of Western Europe (Jones, 1952). Modern science emerged there as a search for relief from desperately bad times, in particular as a reaction to a cruel and repressive religion. The scene was set in Europe with all the players on stage. Christianity had failed them badly with the Black Death, and external elements of science philosophy from China, India, and Arabia played an important role as a stimulus. Today, there are many places where there has never been a Renaissance, i.e., countries with totalitarian political or religious-based power structures. Certainly, none of the Third-World countries have made such a transformation.

We are compelled to pause and ask why so many people are pursuing pure science today. Worldwide, people basically seem to dislike it, but in some places 
it is grudgingly tolerated. Again, why? Many of us scientists are pretty naïve about this, because we love it so much. More succinctly, the celebrated biologist, Harvard's E. O. Wilson, has said, "Man would rather believe than know" (Leakey and Lewin, 1993). Clearly science violates this preference. It has repeatedly exposed facts that most people do not want to see. To this day, the United States has trouble coping with Darwinian evolution.

Of course, the motives for doing science that you hear depend on whom you ask. As examples, we scientists do it because we have an obsessive delight in pursuing our hobbies. America has done it to produce technology to maintain dominance with their military machine and commercial interests. Singapore and Japan do it to strengthen the bases of their industrial machines. The Third World pursues it occasionally in believing it will produce more material wealth, as in First-World countries, and they are sadly mistaken! Regardless of the motives, the current spectacular success of astronomical research in producing scientific knowledge can be neither denied nor ignored.

There are 67 Member Countries in the IAU, and we might benefit by asking which of their observatories have been productive in astronomical research and education, which have not, and why. Certainly, there are wonderful, productive, big, and small observatories today: Cerro Tololo, ESO, AAO, SAAO, Mauna Kea, HST, NASA and ESA probes, and GONG to name a few. Do they have something in common? Are they obviously different from astronomically unproductive countries in Africa, South America, and nearly all of Asia? I believe the successful ones are almost entirely found in cultures which are products of the European Renaissance.

Although the Renaissance was the cradle for the philosophy of modern science, our current paradigm, there have been other productive times and places. During the collapse of intellectual pursuits in Christian Europe in the Dark Ages, astronomy was pursued mostly for religious applications in Muslim societies, countries that are today particularly unproductive scientifically. There are several countries currently making significant investments in installing astronomical observatories primarily on their own - China, South Korea, Egypt, Iran, and especially India (Jayawardhana, 1994) to name a few. Personally, I am pessimistic about these efforts, because I believe that primarily it is the Western countries that have developed societies with the necessary brash attitudes, free thought, and tolerance which are conducive to creative pure science, but we can all hope that with the restructuring of our Global Village at the end of this century and the consequential integration of cultures, they will nevertheless enjoy a degree of success. I believe they would be far more productive if they would at least develop strong partnerships with individual Western observatories.

In debating these issues, one often hears the view that science in developing countries has not proceeded more rapidly because of poor financial backing and a lack of expertise. I disagree with both of these excuses. In fact the lacking of both of these things is a direct reflection of priorities, and except in extreme cases is not a consequence of poor economies. Were the resources that we see devoted in some countries to the military, or to corrupt politicians, or to religious fanaticism, diverted into pursuing astronomy, those countries could afford Keck telescopes. I am not suggesting that such places would be better off pursuing astronomy than their current military, political, and religious hobbies, but it is 
clear that the pursuit of astronomy and other pure sciences is not exactly their preoccupation.

As for creativity and expertise, it would seem that the country that has given Ramanujan and Chandrasekhar to the world is not exactly short on it. From my own personal experiences I have often been impressed by the remarkable amount of local skill one finds in even the most remote and simple villages in undeveloped countries. Nearly always, such people are highly receptive to training and opportunities. That is certainly true of Sri Lanka for example. Rajesh Kochhar of Bangalore, India, has suggested three points in the development of Third-World astronomy with foreign help (Kochhar, 1996):

- training local manpower with expert help from countries with established programmes,

- maintaining and upgrading equipment with foreign help,

- providing a stimulus to sustain the interest.

While Dr. Kochhar's outline succinctly covers the essential points for such developments, I wish he had mentioned the barriers posed by the formidable government bureaucracies invariably found in developing countries, for here only the local nationals are capable of negotiating the nightmares these governments create. Foreigners are helpless to do so. Most of all, I believe he missed the key point: a modern research observatory in the Third World will be far more successful if it has a strong association and partnership with a foreign observatory, in particular one in the West!

Today, in some Third-World countries, highly productive observatories are producing copious data where they are administered from Western countries. In Chile, there is Cerro Tololo, which is headquartered in Arizona, the European Southern Observatory, which is administered by many European countries with headquarters in Germany, and the Las Campanas Observatory, which is administered from Pasadena, California. While the particularly productive South African Astronomical Observatory is currently owned and operated from within that developing country, its international staff is largely composed of British Commonwealth and American imports.

In Sri Lanka there is an entrenched and powerful bureaucracy and attempts to invest there, made by foreign companies, have often met with such inefficiency and corruption that those companies have abandoned their efforts for other shores. At the Arthur Clarke Institute some staff feel discouraged, because they believe an effective astronomical staff cannot be hired within the restrictive hiring criteria imposed upon them. This sad condition will not be corrected any time soon, and it is probably the principal barrier to a partnership with a foreign country, even for a non-commercial and educational facility such as an astronomical observatory.

\section{Conclusions and Summary}

In concluding, I would like to answer "yes" to the interrogative title of this talk. A modern telescope in Sri Lanka and other Third-World countries, could benefit 
those countries and international astronomy significantly when they have a close scientific and administrative relationship with a foreign observatory, hopefully a Western one which provides the scientific leadership. For local young people who want to study science, such observatories offer educational opportunities. For the foreign institutions, it means fascinating data, often obtained from locations with desirable combinations of latitude, longitude, and climate. The resulting science education means an enrichment for a type of education that offers experience in problem solving and deductive reasoning.

It would be a serious mistake to confuse the past and traditional concepts of colonialism with the highly successful Western leadership found operating currently at astronomical observatories in developing countries. We do not find at them patronising attitudes or innuendoes of domination, subjugation, or weak cultures. Although Western leadership at them is a necessary ingredient, we are talking here about collaborations based on mutual respect and benefit. Sir Arthur Clarke recently expressed to me the view that although the well-intended gift of the GOTO telescope has produced negligible results, astronomical telescopes are the most long lived of scientific instruments and that, eventually, when Sri Lanka is again politically stable, this telescope's time will come. Unfortunately, I am unable to share Sir Arthur's prognosis on this point.

Certainly, there are many notable productive old telescopes in use today, just as he has said, but there are also counter examples. One recalls the Jewitt Schmidt telescope at the Harvard College Observatory, which was built on a weakly conceived programme. In the entire lifetime of that instrument, it did not produce a single datum of meaningful astronomical data. The expensive Damon cameras there are another example.

I believe the sad political instability of Sri Lanka is also not relevant. Only when the telescope is associated with a partnership to include leadership from a Western observatory will the project at the ACCIMT stand a chance of developing a meaningful programme. Just as Dr. Wijewardena predicted seven years ago, any attempts to prop up the project with further developments will waste limited resources, until and unless such an administrative restructuring is established.

For Sri Lanka, a continuation by a Western astronomer of the initial site survey by Simon Tulloch would be an essential next step in setting up a productive telescope. Educational centres like the University of Moratuwa have good faculties and talented students who would love nothing more than the challenge of setting up a simple but serious research facility with the guidance of Western astronomers. A modest investment in such a site survey would stimulate the enthusiastic students, who could be employed for many of the activities. If successful, the survey could be followed by using impressive local talent to design and build a small automated telescope, perhaps with further guidance from an institute like the Global Network of Automated Telescopes (GNAT).

I hope my critical remarks today are not discouraging for a country like Sri Lanka, and let me mention a truly striking impression I've had there. In the past few years, it has been my pleasure to participate in seminars on astronomical topics with high-school and university students throughout Sri Lanka, sometimes in rather remote locations. I have been impressed consistently by the enthusiastic 
response I have received from these young students, many of whom would love nothing more than to pursue serious careers in science or engineering.

Finally, let me close with an interesting experience I have recently enjoyed in Sri Lanka. Just a few months ago, I was introduced by Sir Arthur Clarke to an established lawyer in Sri Lanka, Mr. Ranjan Sriskantha, who approached the two of us with a particularly intriguing idea. Mr. Sriskantha has proposed a donation of his property in the highlands near Kandy as a site for an astronomical observatory. Mr. Sriskantha's site may indeed offer a dark-sky location at a moderate altitude, easy access, adequate security, and proximity to a major university, all the things described as impossible at the government meeting seven years ago. Site testing would quickly reveal the realities, but from conversations with Mr. Sriskantha I was struck by something subtle and perhaps more important.

Mr. Sriskantha's enthusiasm for astronomy comes from the private sector in that country. While I do not want to overinterpret this significance, the private sector of any country usually reflects more accurately the intellectual mood than government-administered programmes, and for a country in which innovation is systematically strangled by government bureaucrats, perhaps the private sector may offer efficiency and more responsive activities.

Acknowledgments. I gratefully acknowledge helpful comments from Drs. Philip Catton, Dennis Dutton, Owen Gingerich, Robert Latzer, William Moreau, and Sir Arthur Clarke.

\section{References}

Irwin, John 1952, Science 115, 223.

Irwin, John 1966, Science 152, 1597.

Irwin, John 1966, Science 154, 1275.

Jayawardhana, Ray 1994 Science 264, 502.

Jones, W. T. 1952, Hobbes to Hume, A History of Western Philosophy.

Kochhar, Rajesh 1996, Small Telescopes in Research and Education.

Leakey, Richard and Lewin, Roger 1993, Origins Reconsidered.

Tulloch, S.M. 1996, MNASSA bf 55, 10.

\section{Discussion}

Wentzel commented that the TAD IAU project discussed with the Physics Department of the University of Colombo the possible introduction of some astronomy courses (from visiting professors) in connection with the nearby new telescope. They insisted that physicists active in school astronomy should be trained abroad - impossible for TAD to finance. This suggested to him that there was no real administrative interest at the University.

Narlikar remarked that there has to be a perceived need for a facility before it is donated or created. At IUCAA they began with $35-\mathrm{cm}$ and $40-\mathrm{cm}$ telescopes for Indian universities and these created an awareness of the need for observa- 
tional astronomy in the universities, leading to a proposal for a 2-m telescope, which should be ready in 2001 and will generate considerable research activity.

Editor's note: See the clarification of Japanese procedure for donating telescopes in Kitamura's poster paper, pp. 312-3. For another view of the relative importance of security in decisions concerning sites for an observatory, see the paper by Onuora, p. 329-332.) 